\title{
First-order Reversal Curve Analysis of Kinetic Monte Carlo Simulations of First- and Second-order Phase Transitions
}

\author{
I. Abou Hamad ${ }^{1,2}$, D.T. Robb ${ }^{2}$, P.A. Rikvold ${ }^{1,2,3}$ \\ ${ }^{1}$ Center for Materials Research and Technology and Department of Physics, Florida State University, Tallahassee, FL 32306-4350, USA \\ ${ }^{2}$ School of Computational Science, Florida State University, Tallahassee, FL 32306-4120, USA \\ ${ }^{3}$ National High Magnetic Field Laboratory, Tallahassee, FL 32310
}

February 24, 2019

\begin{abstract}
The dynamics near first- and second-order phase transitions in a two-dimensional lattice-gas model are compared using the first-order reversal curve (FORC) method. The FORC diagram of a first-order transition is characterized by a negative region separating two positive regions, reflecting a competition between the time-varying electrochemical potential and the tendency of the system to phase order.
\end{abstract}

\section{Introduction}

The first-order reversal curve (FORC) method was conceived [1] in connection with the of magnetic systems, as a means to determine the Preisach distribution. Since its initial application to magnetic recording media 2, the FORC method has been applied to a variety of magnetic systems, ranging from magnetic nanostructures to geomagnetic compounds, undergoing rateindependent (i.e., very slow) magnetization reversal. Recently there have also been several FORC studies of rate-dependent reversal [3, 4, 5].

Here we apply FORC analysis to rate-dependent reversal in lattice-gas models of electrochemical deposition. Specifically, we study a two-dimensional 
lattice-gas model with attractive nearest-neighbor interactions, being driven across its first-order phase transition by a time-varying electrochemical potential, as well as a lattice-gas model with repulsive lateral interactions and nearest-neighbor exclusion, being driven across its second-order transition.

For the lattice-gas system, the FORC method consists of saturating the adsorbate coverage in a strong positive electrochemical potential, and, in each case starting from saturation, decreasing the potential to a series of progressively more negative 'reversal potentials' $\bar{\mu}_{r}$, and subsequently increasing the potential back to the saturating value [2]. This produces a family of FORCs $\theta\left(\bar{\mu}_{r}, \bar{\mu}\right)$, where $\theta$ is the adsorbate coverage, and where $\bar{\mu}$ is the instantaneous potential during the increase back to saturation. It is often useful to calculate the FORC distribution,

$$
\rho=-\frac{1}{2} \frac{\partial^{2} \theta}{\partial \bar{\mu}_{r} \partial \bar{\mu}},
$$

which measures the sensitivity of the dynamics to the progress of reversal on the major loop. Note that to normalize the FORC distribution, the

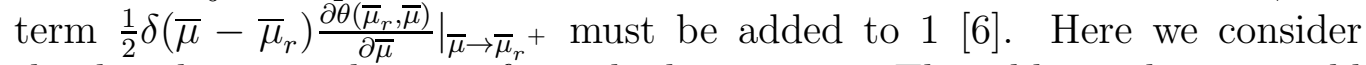
the distribution only away from the line $\bar{\mu}=\bar{\mu}_{r}$. The additional term could be found from the major loop in Figs. 19 and 2a. The FORC distribution is usually displayed as a contour plot called a 'FORC diagram'.

\section{Model}

Kinetic Monte Carlo (KMC) simulations of a lattice-gas model, where a Monte Carlo (MC) step corresponds to an attempt to cross a local freeenergy barrier, have been used to simulate the kinetics of electrochemical systems with first- [7, 8] or second-order [9, 10] phase transitions in two dimensions. Similar to the standard Ising Hamiltonian, a grand-canonical effective lattice-gas Hamiltonian [11, 7, 12, 10, 13.

$$
\mathcal{H}=-\sum_{i<j} \phi_{i j} c_{i} c_{j}-\bar{\mu} \sum_{i=1}^{N} c_{i},
$$

is used to describe the energy associated with a lattice-gas configuration. Here, $\sum_{i<j}$ is a sum over all pairs of sites, $\phi_{i j}$ are the lateral interaction energies between particles on the $i$ th and $j$ th lattice sites, $\bar{\mu}$ is the electrochemical potential, and $N=L^{2}$ is the total number of lattice sites for an $L \times L$ square 
lattice with periodic boundary conditions. The local occupation variable $c_{i}$ is 1 if site $i$ is occupied and 0 otherwise. In addition to adsorption/desorption steps, we include diffusion steps with a comparable free-energy barrier [10].

Independent of the diffusion, attractive nearest-neighbor interactions $\left(\phi_{i j}>\right.$ 0 ) produce a first-order phase transition equivalent to that which occurs below the Curie temperature in the ferromagnetic Ising model. In contrast, repulsive long-range interactions $\left(\phi_{i j}<0\right)$ and nearest-neighbor exclusion produce a second-order phase transition, equivalent to that in an antiferromagnetic Ising model.

\section{First-order phase transition}

Using an $L=128$ lattice-gas model with attractive interactions, a family of FORCs were simulated, averaging over $10 \mathrm{KMC}$ realizations for each reversal curve at room temperature. Using the notation of Ref. [10, the interaction strength (restricted to nearest-neighbor) was taken to be $\phi_{i j}=\phi_{\mathrm{nn}}=55 \mathrm{meV}$. The barriers for adsorption/desorption and diffusion (nearest-neighbor only) were $\Delta_{\mathrm{a} / \mathrm{d}}=\Delta_{\mathrm{nn}}=150 \mathrm{meV}$. The reversal electrochemical potentials $\bar{\mu}_{r}$ associated with the reversal curves were separated by $1 \mathrm{meV}$ increments in the interval $[-200,0]$, and the potential-sweep rate was constant at $|\mathrm{d} \bar{\mu} / \mathrm{d} t|=$ $0.3 \mathrm{meV} / \mathrm{MCSS}$. The FORCs are shown in figure 1 $1 \mathrm{a}$, with a vertical line indicating the position of the coexistence electrochemical potential, $\bar{\mu}_{c}$, and circles showing the positions of the minima.

In a simple Avrami's-law analysis, the FORC minima all lie at $\bar{\mu}=\bar{\mu}_{c}[5]$. However, in the simulations the minima are displaced. For $\theta>0.5$, the minima occur at $\bar{\mu}<\bar{\mu}_{c}$, precisely at the points where the tendency to phase order, which drives local regions of the system toward the nearby metastable state $(\theta \approx 1)$, is momentarily balanced by the electrochemical potential, which drives the system toward the distant stable state $(\theta \approx 0)$. For $\theta<0.5$, the stable and metastable states are $\theta \approx 1$ and $\theta \approx 0$, respectively, and the same analysis explains the FORC minima occurring at $\bar{\mu}>\bar{\mu}_{c}$.

The net effect is a 'back-bending' of the curve connecting the minima, as seen in Fig. 17. The definition in Eq. 1 implies that the FORC distribution $\rho$ is negative in the vicinity of the back-bending as seen in Fig 10, where the FORC distribution is plotted against the variables $\bar{\mu}_{b}=\left(\bar{\mu}_{r}+\bar{\mu}\right) / 2$ and $\bar{\mu}_{c}=$ $\left(\bar{\mu}_{r}-\bar{\mu}\right) / 2$. The negative values of $\rho$ reflect a local divergence of the FORCs, which can be considered a dynamical instability, caused by the competition 

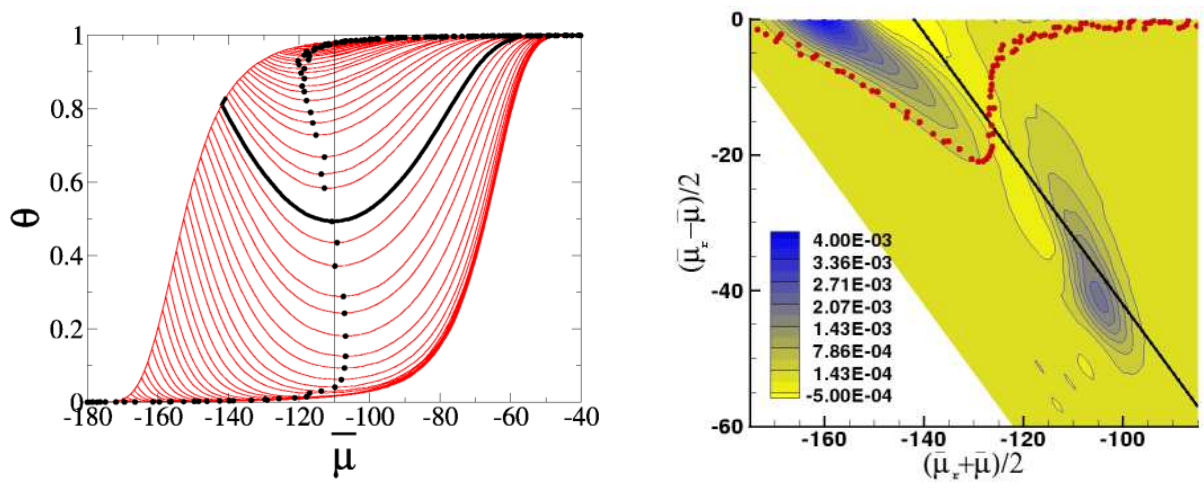

Figure 1: (a) First-order reversal curves (FORCs) of a first-order phase transition. The vertical line shows the position of the coexistence value $\bar{\mu}=\bar{\mu}_{c}$. The minima of each FORC are also shown (circles). (b) FORC diagram generated from the family of FORCs shown in (a). The positions of the FORC minima are also shown (circles). The straight line corresponds to the FORC for which the minimum lies at the coexistence value $\bar{\mu}=\bar{\mu}_{c}$.

between the tendency to phase order and the effect of the potential. It is interesting to note that the curve connecting the minima of the FORCs resembles a van der Waals loop, but with an asymmetrical shape about the point $\left(\bar{\mu}=\bar{\mu}_{c}, \theta=0.5\right)$, and with a sweep-rate dependent shape (not shown). We plan to explore the connection with the van der Waals loop in simulations of a mean-field model in future work.

\section{Second-order phase transition}

Using the same Hamiltonian, but with long-range repulsive interactions and nearest-neighbor exclusion, KMC simulations were used to produce the FORCs for a second-order phase transition. The reversal potentials $\bar{\mu}_{r}$ were separated by $10 \mathrm{meV}$ increments in the interval $[-200,400]$. As in Ref. [10], the repulsive $1 / r^{3}$ interactions, with nearest-neighbor exclusion and $\phi_{\mathrm{nnn}}=-21 \mathrm{meV}$, are calculated with exact contributions for $r_{i j} \leq 3$, and using a mean-field approximation for $r_{i j}>3$. The barriers for adsorption/desorption, and nearestand next-nearest-neighbor diffusion, are $\Delta_{\mathrm{a} / \mathrm{d}}=300 \mathrm{meV}, \Delta_{\mathrm{nn}}=100 \mathrm{meV}$, 

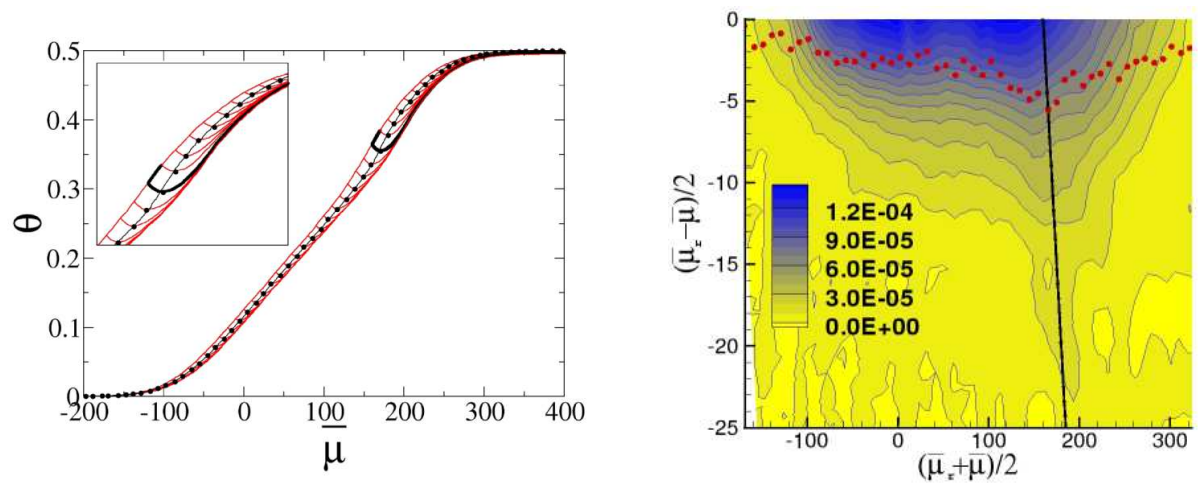

Figure 2: (a) FORCs for a second-order phase transition. The black middle line shows the equilibrium curve. The inset is a magnification of the critical region. The minima of each FORC are also shown (black circles). (b) FORC diagram generated from the FORCs shown in $(\mathbf{a})$. The positions of the FORC minima are also shown (circles). The straight line corresponds to the FORC for which the minimum lies closest to the critical coverage.

and $\Delta_{\text {nnn }}=200 \mathrm{meV}$. A second-order phase transition occurs between a disordered state at low coverage and an ordered state at high coverage [9, 10]. The FORCs and FORC diagram are shown in figure 2. Also indicated in Figure $2 \mathrm{a}$ are the FORC minima and the equilibrium isotherm.

An important distinction here is that the FORC minima lie directly on the equilibrium isotherm. This is because such a system has one stable state for any given value of the potential, defined by the continuous equilibrium curve. The positive values of the FORC distribution in Fig. 20 are consistent with relaxation toward this equilibrium curve, at a rate which increases with the distance from equilibrium. It is interesting to note that, while it is difficult to see in Fig. $2 a$, the rate of approach to equilibrium decreases greatly along the first FORC that dips below the critical coverage $\theta_{c}=0.36$ (shown in bold in Fig. 2a). This is clearly revealed in the FORC diagram in Fig. 2b, where it is reflected by the Florida-shaped "peninsula" centered around this FORC. 


\section{Comparison and conclusions}

Two observations can be made by comparing the FORCs and FORC diagrams for systems with first- and second-order phase transitions. First, the FORC minima in systems with a second-order phase transition correspond to the equilibrium behavior, while for systems with a first-order phase transition they represent van der Waals-like metastable and unstable regions. Thus, FORCs can be used to recover equilibrium behavior for systems with a second-order transition which need a long time to equilibrate. Second, due to the instability that exists in systems with a first-order phase transition, the corresponding FORC diagram contains negative regions which do not exist for systems with a second-order phase transition.

\section{Acknowledgments}

This research was supported by U.S. NSF Grant No. DMR-0240078, and by Florida State University through the School of Computational Science, the Center for Materials Research and Technology, and the National High Magnetic Field Laboratory.

\section{References}

[1] I. D. Mayergoyz: IEEE Trans. Magn. MAG 22, 603 (1986).

[2] C. R. Pike, A. P. Roberts, K. L. Verosub: J. Appl. Phys. 85, 6660 (1999).

[3] C. Enachescu, R. Tanasa, A. Stancu, F. Varret, J. Linares, E. Codjovi: Phys. Rev. B 72, 054413 (2005).

[4] M. Fecioru-Morariu, D. Ricinschi, P. Postolache, C. E. Ciomaga, A. Stancu, L. Mitoseriu: J. Optoelectron. Adv. Mater. 6, 1059 (2004).

[5] D. T. Robb, M. A. Novotny, P. A. Rikvold: J. Appl. Phys. 97, 10E510 (2005).

[6] C. R. Pike: Phys. Rev. B 68, 104424 (2003).

[7] S. Frank, D. E. Roberts, P. A. Rikvold: J. Chem. Phys. 122, 064705 (2005). 
[8] S. Frank, P. A. Rikvold: Surf. Sci. (2006), in press.

[9] S. J. Mitchell, S. Wang, P. A. Rikvold: Faraday Disc. 121, 53 (2002).

[10] I. Abou Hamad, P. A. Rikvold, G. Brown: Surf. Sci. 572, L355 (2004).

[11] C. N. Yang, T. D. Lee: Phys. Rev. 87, 410 (1952).

[12] I. Abou Hamad, Th. Wandlowski, G. Brown, P. A. Rikvold: J. Electroanal. Chem. 554-555, 211 (2003).

[13] I. Abou Hamad, S. J. Mitchell, Th. Wandlowski, P. A. Rikvold: Electrochim. Acta 50, 5518 (2005). 